\title{
Servicios educativos y sanitarios elementales en el medio rural: percepción social e influencia sobre la calidad de vida
}

\section{Basic education and health-care services in rural areas: social perception and influence on the quality of live}

\author{
Jaime Escribano Pizarro*
}

\section{CALIDAD DE VIDA EN El MEDio RURAL}

Hasta hace poco, el análisis geográfico del medio rural se apoyaba casi por completo en estudios etnográficos sobre la evolución y el cambio acontecido en las relaciones económicas y políticas de este espacio. Sin embargo, la creciente realización de trabajos sociales y culturales sobre la población rural ha alterado esta tendencia. Ahora son más habituales las investigaciones sobre los discursos e interpretaciones que los habitantes de zonas rurales realizan de su vida diaria, lo que permite avanzar con mayor facilidad en la comprensión de los procesos de producción del espacio rural — como la contraurbanización, la gentrificación, etc.- . Procesos en los que cada vez resultan más determinantes las interpretaciones que realiza la población, sobre aspectos como la ruralidad y la calidad de vida del medio rural (Brereton et al., 2011, p. 204).

De hecho, son varias las investigaciones que señalan la elevada calidad de vida de que disfruta la población de zonas rurales, especialmente si las comparamos con las urbanas (Richmond et al., 2000, pp. 159-160; Pospěh, Delín y Spěšná, 2009, p. 292; Shucksmith et al., 2009, pp. 1.283-1.285). Sin embargo,

\footnotetext{
* Instituto Interuniversitario de Desarrollo Local, Universidad de Valencia (jaime.escribano @uv.es).
} 
esto no significa que la sociedad rural sea en general optimista sobre su futuro; más bien al contrario, ya que observa como los cambios que se producen en su espacio no están protagonizados por ella, ni responden a proyectos propios. Básicamente, porque cualquier alternativa de futuro parece asentarse en una percepción urbana de la ruralidad, apoyada en estilos de vida y valores tradicionales, y en donde la calidad de vida se concibe, primero, como oposición a todo cambio que se aleje de un pasado idílico; y segundo, de forma subyacente, como rechazo al actual estilo de vida urbano (Ray y Ward, 2006, pp. 5 y 8; Bustos, 2009, pp. 455-457).

En consecuencia cuando desde las ciudades se habla de calidad de vida, más valorados son aspectos propios de zonas rurales como la disponibilidad de espacio abierto de carácter privado — para la construcción de viviendas de baja densidad — y/o colectivo — para la realización de actividades de ocio, deportivas, etc.—; la proximidad a un medio físico único, singular, y «poco» alterado, en donde prevalezcan los espacios verdes y sea posible un contacto rápido y directo con la naturaleza; la mayor sensación de seguridad, ante problemas derivados del exceso de circulación, conductas delictivas, etc.; ciertos atractivos económicos, como por ejemplo el menor coste del metro cuadrado; las mayores calidades de los recursos ambientales — como el aire y el agua-y alimentarios - productos naturales y frescos, generados en circuitos cortos-; las relaciones sociales de proximidad caracterizadas por valores de amistad, sinceridad, confianza... (Richmond et al., 2000, p. 162; Deller et al., 2001, pp. 352-354; Auh y Cook, 2009, pp. 377-378 y 387-388; Grgić et al., 2010, pp. 655-658; Brereton et al., 2011, p. 223).

Ahora bien, hablar de calidad de vida en zonas rurales es algo más complejo, ya que no hay una única definición aceptada y consensuada del propio concepto de calidad de vida. En general se trata de una noción que difiere de una persona a otra, de manera que resulta complicado establecer con precisión cuántas y qué variables considerar, su carga de subjetividad, y las variaciones que pueden presentar según el tipo de sociedad en la que trabajemos. En consecuencia, la mayoría de autores coinciden en que se trata de un concepto al que cabe aproximarse desde una triple perspectiva (Massam, 2002, pp. 145-157; Royuela, Lambiri y Biagi, 2008; Gómez Piñeiro, 2009, pp. 284-286; Kazana y Kazaklis, 2009, pp. 210-211; Pospěh, Delín y Spěšná, 2009, pp. 285-286):

a) Según la diferenciación entre una dimensión psicológica —cultural o subjetiva - y otra ambiental - material u objetiva-. La primera hace referencia a toda una serie de mecanismos psicológicos del ser humano, que determinan el grado de satisfacción con que cada persona califica sus experiencias vividas — grado de libertad, confianza, felicidad, etc.—. Por su parte, la se- 
gunda alude a las condiciones externas que activan esos mecanismos internos; a menudo, se trata de una dimensión asociada a espacios concretos de vida, como por ejemplo la configuración del lugar de residencia, la calidad ambiental del mismo, etc., relacionados a su vez con el entorno más inmediato, es decir, con el territorio en su vertiente local. Recientemente, esta escala espacial habría modificado su perspectiva gracias al aumento de la movilidad, y al uso constante y continuo de las tecnologías de la información y la comunicación (TIC). En todo caso, la calidad de vida es un conjunto de múltiples facetas en donde resulta clave combinar las dos dimensiones para obtener una imagen completa de la calidad de vida de cada persona y/o de cada espacio.

b) En función de la adopción de una visión individual o colectiva, resultado esta última de la pertenencia a una sociedad o a un grupo específico de ella. La calidad de vida puede definirse como la satisfacción o felicidad individual con la vida y el medioambiente, incluyendo en ésta necesidades y deseos, aspiraciones, preferencias por estilos de vida concretos, etc. Pero también puede concebirse como el resultado de las interrelaciones sociales y medioambientales de las que se dispone a nivel colectivo, y de los aspectos económicos y culturales que guían al grupo o sociedad al que se pertenece.

c) Y por último, la calidad de vida se refiere al mayor o menor grado de satisfacción que cada persona alcanza a lo largo de su vida. Es decir, no es un concepto estático en el tiempo, sino que se trata de un proceso que se va construyendo de manera continua, de forma que se pueden realizar comparaciones en diferentes momentos.

Ahora bien, si regresamos al conjunto de aspectos que favorecen la calidad de vida en el medio rural, llama la atención que entre los elementos señalados con mayor regularidad no aparezcan ni los servicios básicos educativos, ni los sanitarios. Un hecho paradójico si tenemos en cuenta la revalorización que los servicios públicos en general - especialmente estos dos-, alcanzan hoy en día en numerosas zonas rurales. Primero, como consecuencia del reciente viraje neoliberal del Estado de Bienestar; y segundo, por el aumento de las demandas de una población rural más plural y «numerosa».

En primer lugar, la desregularización y privatización de industrias y servicios hasta hace poco nacionales, como bancos, eléctricas, empresas de telecomunicaciones, de transporte público, hospitales, etc., ha reducido de forma considerable el papel del Estado en la prestación de servicios. Es cierto que estos cambios no se limitan a zonas rurales, pero sus impactos son mayores en ellas debido a que sus bajas densidades y elevadas dispersiones de población, incrementan las dificultades de mantener activos servicios de este tipo. De hecho, aunque son numerosas las administraciones que intentan garantizar una 
protección mínima, la mayoría termina por adoptar prácticas de mercado en la ordenación y en la gestión de estos servicios, como por ejemplo mediante la concentración de las prestaciones en unidades de mayor tamaño - con el cierre correspondiente de los servicios poco o nada rentables económicamente-, o la fijación de precios según criterios geográficos (Furuseth, 1998, pp. 236-238; Woods, 2006, pp. 584-585).

En segundo lugar, los servicios educativos y los sanitarios han aumentado su valor en el medio rural debido a que forman parte de los aspectos sociales que más urgentemente necesitan mejorarse, para atender así a un conjunto de población cada vez «numeroso» y exigente —en cuanto a calidad y cantidad de ofertas- (Michalski y Marland, 2001, pp. 10-14; Bustos Gisbert, 2005, pp. 145-146; Fundación Encuentro, 2007, pp. 373-387; Grgi et al., 2010, pp. 658-660; Brereton et al., 2011, p. 220). Sobre todo por la diversidad de su composición, ya que junto a los residentes tradicionales nos encontramos cada vez más con un mayor número y tipo de habitante rural, y por tanto de demandas y necesidades - particularmente, procedentes de inmigrantes urbanos de zonas cercanas-.

Así, mientras que la población local busca evitar, o simplemente mitigar, el desmantelamiento gradual que presenta gran parte del espacio rural en materia de infraestructuras básicas —no sólo relacionadas con la educación y la sanidad, también con los transportes, las telecomunicaciones, la vivienda, el empleo, etc.- los recien llegados esperan encontrar unos niveles de atención similares a los de sus zonas urbanas de procedencia, de forma que difícilmente acepta ciertas carencias en las prestaciones básicas. Sobre todo en aquéllas para las que se encuentran más «desprotegidos», ante la falta de medios tradicionales mediante los que dar respuesta a sus demandas - como es el papel de la familia en el cuidado de niños y/o adultos con necesidades especiales(Brereton et al., 2011, pp. 221-222).

En este contexto, resulta interesante determinar cómo los servicios y los equipamientos educativos y sanitarios de nivel elemental participan en la valoración que realiza la población rural sobre su calidad de vida. En especial, para conocer si hay o no algún tipo correlación entre ésta y la influencia que en ella pueden ejercer las prestaciones vinculadas a ambos servicios. Es decir, si la oferta educativa y sanitaria local aparece: a) como un componente explícito de la calidad de vida a partir de la que se puede obtener cierta satisfacción y/o bienestar personal; b) como un medio para alcanzar y/o beneficiarse de una serie de factores que serán los que permitan hablar realmente de calidad de vida; o c) como un conjunto de elementos que no intervienen en la valoración, más o menos positiva, con que cada individuo percibe su calidad de vida. 
Metodología: Sujetos y ÁREA DE ESTUdio, RECOGIDA Y ANÁLISIS DE DATOS

La evaluación de la calidad de vida se ha realizado tradicionalmente desde una doble perspectiva: por un lado, bajo un enfoque cuantitativo, objetivo y medible con relativa facilidad, a través de estudios apoyados en indicadores cuantificables de corte socioeconómico destinados por ejemplo, a determinar el grado en el que se cubren las necesidades humanas — como el Índice de Desarrollo Humano-; por otro, desde una visión subjetiva, basada en el conocimiento de la apreciación que las personas tienen sobre su calidad de vida, sobre sus necesidades. Obviamente, este segundo enfoque está condicionado no sólo por la población que juzga su bienestar, sino también por aquélla con la que se compara.

Sin embargo, en la medida que trabajamos sobre territorios, y estos evolucionan y desarrollan nuevas complejidades, el enfoque cualitativo aparece como relativamente más sugerente. En particular, porque junto a las medidas económicas tradicionales, también es necesario conocer y evaluar de forma directa la percepción y las características cambiantes de los individuos en relación a sus redes sociales, acceso y uso de los servicios, y las dimensiones medioambientales de su calidad de vida (Brereton et al., 2011, p. 205). Y porque una cosa es la imagen que ofrece un territorio, y otra distinta la que perciben sus habitantes (Gómez Piñeiro, 2009, p. 293).

Ahora bien, en el medio rural la tarea de analizar la calidad de vida se hace aún más compleja no sólo por la falta de datos directos y actuales, sino también por la diversidad de situaciones que caracterizan a los espacios rurales —algunos territorialmente más dinámicos que otros-, lo que sin duda influye en la valoración que la población rural tiene sobre su calidad de vida. Por ello, a continuación adoptamos una metodología cualitativa, y tomamos con unidad de análisis espacial el territorio rural de la provincia de Valencia delimitado por la iniciativa comunitaria LEADER+ (2000-2006). Éste, pese a su relativo dinamismo, engloba todo un conjunto de poblaciones capaces de ejemplificar gran parte del cambio territorial acontecido en los espacios rurales españoles durante las últimas décadas (Piqueras, 2012, pp. 71-78; Esparcia y Noguera, 2001, pp. 345-356), y por tanto, poder mostrarnos no sólo qué aspectos condicionan la calidad de vida en medio rural, sino también cómo lo hacen.

Para ello, en concreto utilizamos como casos de estudio una serie de municipios capaces de recoger tanto los contrastes territoriales del medio rural como, en función del objetivo planteado, las diferencias socioespaciales que la organización funcional de los servicios básicos de educación y sanidad generan sobre el territorio. Para el primer aspecto nos hemos basado en el tamaño demográfico municipal, puesto que a priori cuanta mayor población, mayor es también la po- 


\section{FIGURA 1}

\section{MUNICIPIOS DE ESTUDIO: EVOLUCIÓN DEMOGRÁFICA 2001-2010 Y TAMAÑO DE POBLACIÓN 2010}
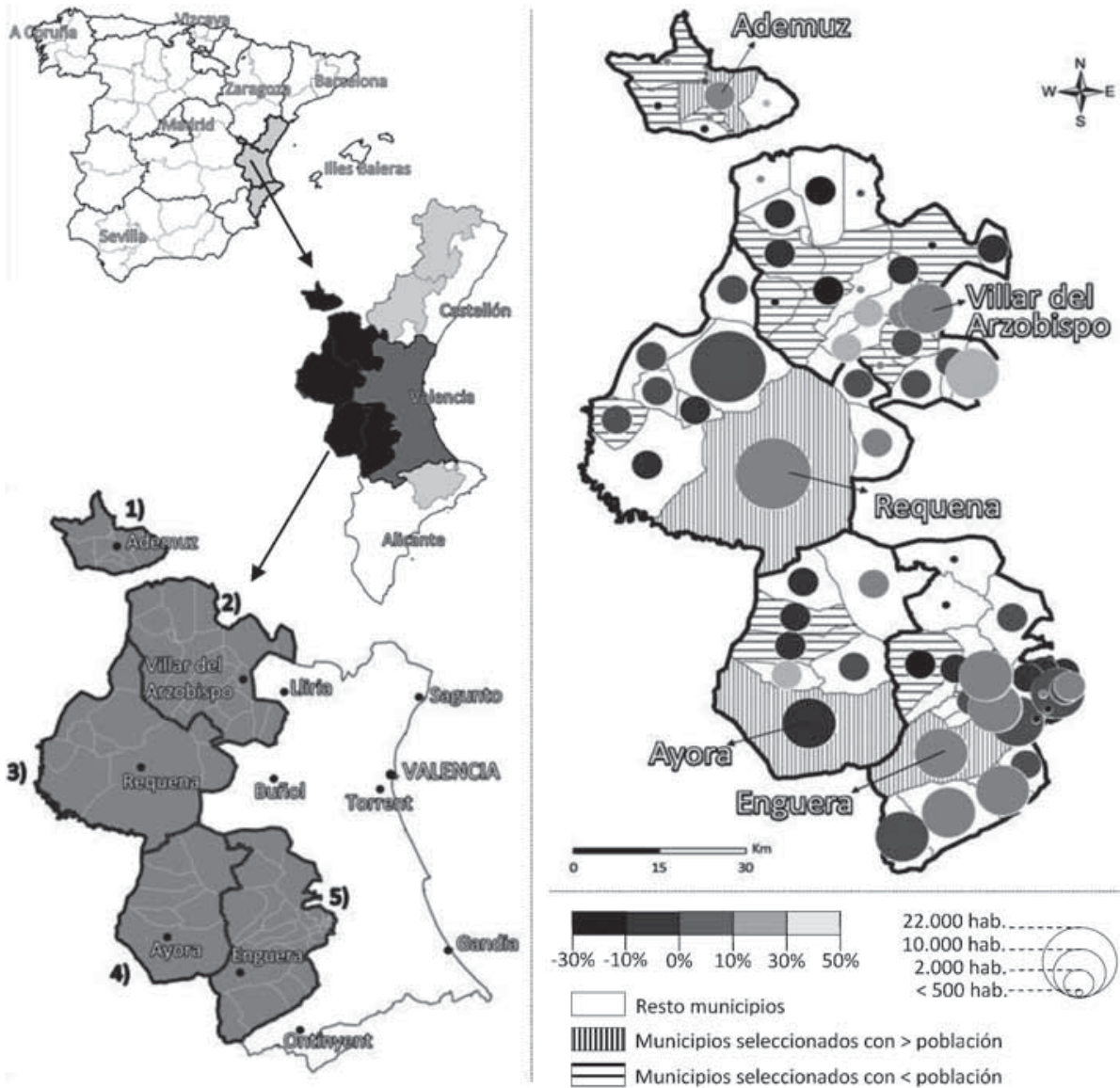

Nota: en la provincia de Valencia podemos distinguir: 1) Rincón de Ademuz; 2) Serranía del Turia; 3) Tierras del Interior; 4) Valle de Ayora-Cofrentes; 5) Macizo del Caroig. El resto de grupos LEADER+ de la Comunidad Valenciana se reparten, como se puede ver en el mapa autonómico, dos en la provincia de Castellón (al norte Els Ports-Maestrat, y al sur de éste limitando con la provincia de Valencia el Alto Palancia - Alto Mijares) y uno más en la provincia de Alicante (Aitana).

Fuente: elaboración propia a partir de la Unidad de Promoción y Animación del Desarrollo Rural y los Grupos de Acción Local de los LEADER+ elegidos, los Censos de Población y Viviendas de 2001 y el Padrón Municipal de 2010 del INE (2011).

Estudios Geográficos, Vol. LXXIII, 272, pp. 35-61, enero-junio 2012 ISSN: 0014-1496, eISSN: 1988-8546, doi: 10.3989/estgeogr.201202 
sibilidad de estar ante un territorio relativamente más dinámico —al ser posible contar con más iniciativas de todo tipo, mayor participación, etc.—. Y viceversa, los municipios con menor población suelen presentarse regularmente como ejemplo de territorios con escaso dinamismo debido a su «reducido» atractivo económico, geográfico y social para conseguir mantener y/o atraer población (Conselleria de Agricultura, Pesca y Alimentación, 2009, p. 188) (figura 1).

Además, este criterio permite también distinguir con facilidad las valoraciones que la población del medio rural puede tener sobre cómo los servicios básicos señalados influyen en su calidad de vida habitual. En general, porque la actitud de la población no es la misma cuando las prestaciones educativas y las sanitarias están físicamente presentes a nivel local —es decir, núcleos de tamaño demográfico elevado-, o por el contrario, hay que recurrir a instalaciones situadas en zonas próximas ante su inexistencia local, debido a una escasa demanda municipal capaz de rentabilizar económicamente este tipo de prestaciones - por tanto, núcleos con poca población-.

En segundo lugar, las diferencias territoriales que genera la organización funcional de los servicios básicos educativos y sanitarios, se han recogido a través de la distinta tipología y combinación local que presentan sus establecimientos en el espacio rural ${ }^{1}$; básicamente, porque ni todos los municipios disponen simultáneamente de ambos servicios, ni en caso de ser así, presentan el mismo grado de cobertura temporal, igual cartera de prestaciones, o idénticos recursos materiales y/o humanos. En consecuencia, este criterio ha permitido identificar municipios tanto con distintos tipos de oferta educativa y sanitaria local, como caracterizados por presentar únicamente la oferta de alguno de ellos (figura 2).

Finalizada la selección de municipios, la información cualitativa derivó de varias entrevistas personales con diversos actores clave. Estos se eligieron en función de su doble capacidad para, por una parte, ofrecer una visión integral de las diferentes dinámicas demográficas presentes en el espacio rural seleccionado, y vinculadas en modo alguno con la posibilidad de disfrutar de una mayor o menor calidad de vida en éste. Y por otra, generar valoraciones argumentadas sobre el papel de estos en la calidad de vida local, gracias a su vinculación personal y/o profesional con los servicios educativos y/o sanitarios del ámbito elegido.

${ }^{1}$ El sistema valenciano de educación básica distingue los Centros de Educación Infantil y Primaria, y los Colegios Rurales Agrupados. Mientras, el sistema sanitario elemental distingue: Centros de Salud — centros de referencia dotados de servicio de urgencia-; Consultorios Médicos — subordinados funcionalmente a los anteriores—; y Consultorios Auxiliares — sirven de apoyo a las unidades precedentes con una menor dotación de recursos-. 
FIGURA 2

\section{LOCALIZACIÓN Y OFERTA DE SERVICIOS EDUCATIVOS Y SANITARIOS BÁSICOS EN EL ÁREA DE ESTUDIO}

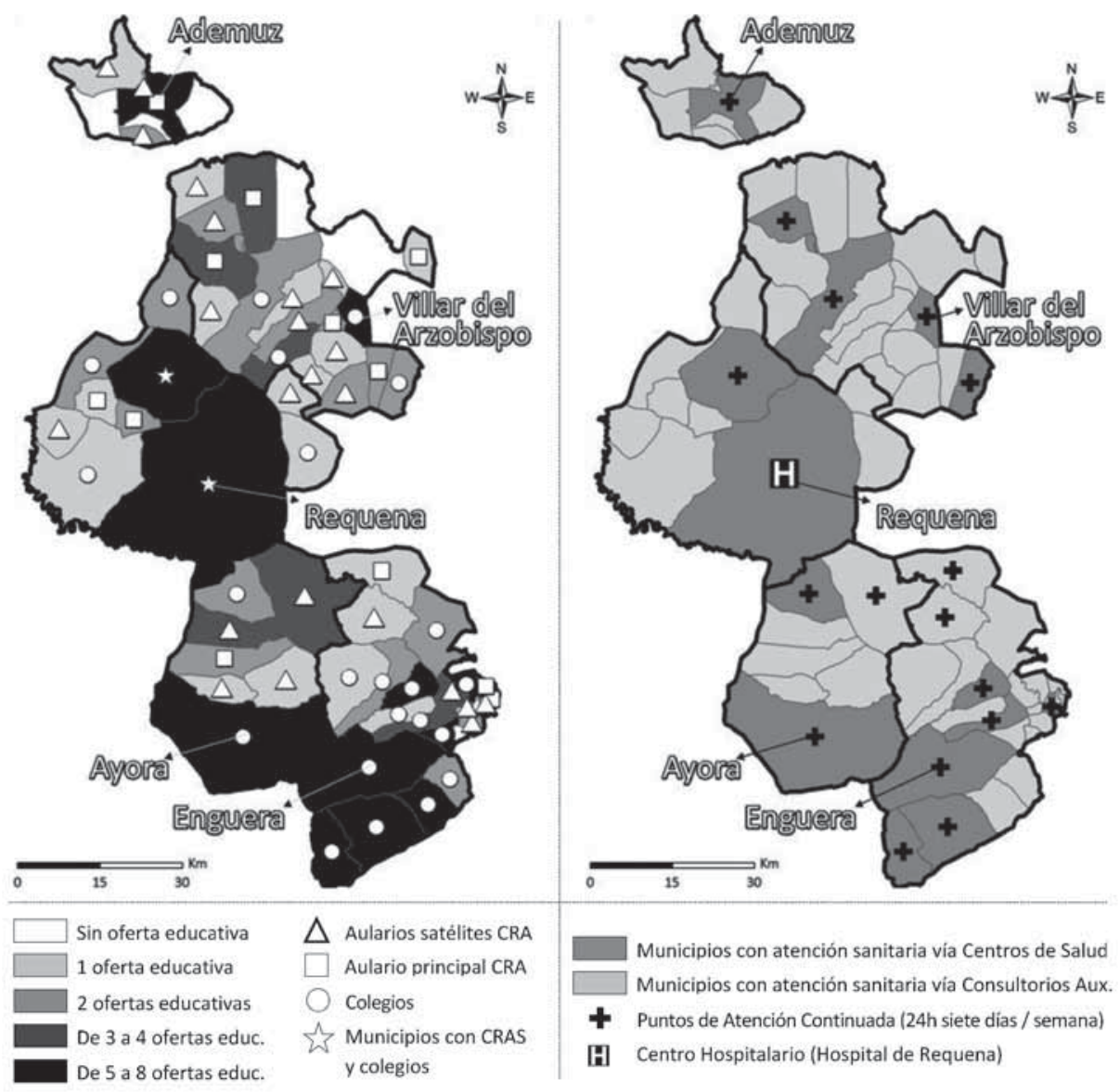

Nota: al hablar de oferta educativa entendemos opciones de educación que integran no sólo el nivel básico, sino también la educación postobligatoria -bachilleratos y módulos de formación profesional-, educación infantil y pre-escolar, escuelas de música, idiomas, centros para adultos, etc. Recordemos que CRA equivale a decir Centro -de educación- Rural Agrupado.

Fuente: elaboración propia a partir de la Guía de Centros Docentes de la Conselleria de Educació (Generalitat Valenciana) y del Listado de Centros de Atención Primaria de la Conselleria de Sanitat (Generalitat Valenciana).

Estudios Geográficos, Vol. LXXIII, 272, pp. 35-61, enero-junio 2012 ISSN: 0014-1496, eISSN: 1988-8546, doi: 10.3989/estgeogr.201202 
De este modo entre los meses de abril y mayo de 2006, y mayo y julio de 2007 , se registraron en audio un total de ochenta entrevistas ${ }^{2}$, cada una de ellas con una duración media comprendida entre los 45 y 60 minutos. Diecinueve a representantes políticos locales y/o supramunicipales; diecinueve más a técnicos encargados de la puesta en marcha y/o la gestión de planes de desarrollo territorial; diecisiete a diversos responsables de establecimientos sanitarios; doce entrevistas a directores y jefes de estudio de centros educativos; y por último, trece más a varios miembros de la sociedad civil encargados de asociaciones relacionadas con el territorio, la sanidad, y/o la educación - presidentes de Asociaciones de Madres y Padres de Alumnos (AMPAS), directores de Escuelas para Adultos, coordinadores de Protección Civil,...-.

Es cierto que la elección de la entrevista personal como método de trabajo principal $^{3}$, y en concreto la de tipo semiestructuado, conlleva tener presentes diversas cuestiones que pueden debatir su validez y consistencia (Valles, 2002, pp. 53-134). De entre ellas, tras nuestro trabajo de campo, destacamos las estrategias utilizadas con las que hacer frente a las dos más habituales.

Por un lado, ante la dificultad de conseguir un registro en audio útil, debido a que el entrevistado se sintiera invadido en su intimidad — personal y/o profesional- o inseguro ante el posible uso posterior de la conversación registrada, se trabajaron especialmente las fases de concertación y presentación. Para ello, se subrayó en cada una de ellas y de forma clara aspectos como el anonimato y la confidencialidad del entrevistado, el objeto y el marco de la investigación, los motivos de su elección, y los beneficios que reportaba su participación como informante clave. Lógicamente, aún así cabe reconocer que en ocasiones las contestaciones ofrecidas se desviaban relativamente de las cuestiones planteadas, abordando temas considerados menos controvertidos y/o de mayor interés por parte del entrevistado; en este sentido, la capacidad de reconducir la conversación mediante reafirmaciones, recapitulaciones, aclaraciones, etc., fue esencial. De este modo, se consiguió que las respuestas

${ }^{2}$ Esta muestra forma parte del conjunto total de entrevistas realizadas para la elaboración de una Tesis Doctoral —financiada la Universidad de Valencia_, enfocada a comparar el papel de los servicios básicos educativos y sanitarios en los procesos de desarrollo local de diferentes áreas rurales de España y de Francia.

${ }^{3}$ Para conseguir mayor información, esta técnica se acompañó en menor medida de varios métodos cualitativos complementarios, como la observación, el desarrollo de conversaciones informales, la realización personal e interpretación de fotografías de los espacios visitados y el uso de fuentes indirectas de tipo documental — prensa nacional y/o regional, boletines de información local, revistas escolares, programaciones de AMPAS, etc.- . 
fueran en su mayoría oportunas y denotasen implicación, reduciendo los tradicionales sesgos derivados de la deseabilidad social.

Y por otro, en tanto en cuanto la entrevista es una herramienta de trabajo susceptible de quedar con facilidad expuesta a errores de traducción y de interpretación de la información por parte del entrevistador, frente a otros métodos como la encuesta estadística, se optó por llevar a cabo un amplio proceso de precodificación temática del objeto de estudio. Para ello, en primer lugar y tras la revisión bibliográfica oportuna, se estructuró el conjunto de la investigación en una serie de temas centrados en aspectos básicos como por ejemplo, la calidad de vida y la calidad de los servicios educativos y sanitarios, la influencia de estos en la evolución sociodemográfica del medio rural, etc. En segundo lugar, se establecieron diferentes categorías de análisis para cada una de las temáticas identificadas, buscando así señalar los aspectos clave que influyen en ellas: decisiones de localización residencial, uso de los equipamientos y prestaciones educativas y/o sanitarias, impacto de los transportes públicos, etc. Y por último, en tercer lugar, se confeccionaron los discursos resultantes con los que responder al objetivo de la investigación, tras relacionar las respuestas de los entrevistados — reagrupándolas por categorías y temas-, con los diferentes procesos territoriales presentes en los dos tipos de municipios-casos de estudio examinados — según su tamaño demográfico—. De hecho, se optó por la entrevista como método de trabajo por la sencillez con que permitía establecer dicha interrelación, al ser una herramienta capaz de recoger con facilidad no sólo información comparable sobre un aspecto tan complejo como es la calidad de vida, sino también el valor argumentado que la sociedad rural otorga al papel de los servicios educativos y sanitarios sobre su bienestar diario.

PERCEPCIÓN SOCIAL SOBRE LA EVOLUCIÓN DE LOS SERVICIOS BÁSICOS EDUCATIVOS Y SANITARIOS EN EL MEDIO RURAL

Antes de analizar si los servicios educativos y sanitarios básicos influyen sobre la calidad de vida en el medio rural, y de ser así, cómo lo hacen, resulta interesante contextualizar la valoración que la sociedad de estas áreas tiene sobre los mismos. Porque pese a la necesidad de continuar con la mejora de las prestaciones asociadas a estos servicios — tal como afirmábamos con anterioridad—, no se puede negar que ambos han experimentado una mejoría notable en las últimas décadas. Sobre todo, como resultado del esfuerzo normativo realizado por las administraciones para mejorar e incrementar la oferta de servicios de todo tipo en zonas rurales, y reducir así las desigualdades cuantitativas y cualitativas con las áreas urbanas (Fundación Encuentro, 2007, pp. 376 y 381-382). 
Por ejemplo, en materia educativa dos son las referencias normativas básicas que ayudan a comprender la valoración positiva que la sociedad rural tiene actualmente sobre la oferta disponible. El Real Decreto 1174/1983 sobre educación compensatoria ${ }^{4}$ es la primera de ellas; básicamente, porque favoreció una transformación cuantitativa y cualitativa sin precedentes para la escuela «rural», al resolver el problema que hasta entonces había generado la aplicación de Ley General de Educación de 19705: el cierre de numerosas escuelas «rurales» ante la supuesta mejora de la calidad educativa que ofrecían los centros comarcales o de concentración (Corchón, 2000, pp. 13-39).

La segunda referencia deriva de la creación de los Colegios Rurales Agrupados $(\mathrm{CRAS})^{6}$, y su progresiva generalización por el espacio rural. Gracias a ellos, se consiguen unificar administrativamente diferentes escuelas «rurales» en una sola, manteniendo en cada municipio los distintos aularios que pudieran existir. De este modo, una cantidad considerable de localidades consigue mantener su escuela municipal, al superarse con esta organización los problemas derivados de cumplir estrictamente con los criterios reglamentarios generales para la «adecuada» prestación educativa —normalmente, de carácter urbano-, como por ejemplo el número de alumnos por aula, los ratios de profesorado, los apoyos de especialistas, etc.

Aparte, lógicamente, esta nueva forma de trabajo agrupado permite que los diferentes equipos directivos y consejos escolares disgregados hasta entonces por el espacio rural, puedan compartir todo tipo de labores y funcionar de este modo como unidades integrales. En consecuencia, no sólo se realizan de forma más sencilla tareas como la asignación presupuestaria, la distribución de materiales y recursos humanos, ... sino que incluso mejoran respecto a situaciones precedentes, ya que al dejar de estar fragmentada la demanda, se consigue rentabilizar mejor cualquier tipo de inversión.

En todo caso, parte de los cambios normativos acontecidos en los últimos años destacan no sólo por solucionar carencias y/o aspectos problemáticos procedentes de situaciones previas, sino también por corregir errores u omisiones que medidas relativamente recientes — con objetivos a priori igual-

${ }^{4}$ RD 1174/1983, de 27 de abril, sobre educación compensatoria. BOE n. 12, de 11 de mayo de 1993.

${ }^{5}$ Ley 14/1970, de 4 de agosto, General de Educación y Financiamiento de la Reforma Educativa. BOE n. 187, de 6 de agosto de 1970.

${ }^{6}$ Orden de 15 de mayo de 1997, de la Conselleria de Cultura, Educación y Ciencia, por la que se regula la constitución de Colegios Rurales Agrupados de Educación Infantil y Primaria en la Comunidad Valenciana. DOGV n. 3.028, de 04 de julio de 1997. 
mente positivos-, han terminado por generar al llevarse a la práctica. En concreto, porque en muchos casos se ha intentado sin éxito compaginar la mejora y el aumento de las dotaciones educativas en las zonas rurales, con las limitaciones económicas resultantes del viraje neoliberal del Estado.

Así ocurre por ejemplo, con la Ley 1/1990 de Ordenación General del Sistema Educativo y su ampliación de la edad escolar obligatoria ${ }^{7}$. Con independencia de los beneficios educativos y los cambios que supuso en la estructura del mercado laboral —la entrada a éste se retrasa hasta los 16 años-, para el territorio rural representó una nueva concentración espacial de la demanda educativa, si bien a una edad más tardía ${ }^{8}$. De hecho, para muchos entrevistados esta diferencia constituye la razón por la que el medio rural no perdió nuevamente parte de su ya reducida estructura educativa elemental, puesto que en comparación fueron menos los centros obligados a cerrar en este momento. Aunque en realidad, como también se reconoce, el problema fundamental seguía existiendo, ya que sólo se conseguía desplazar en el tiempo la salida de sus poblaciones más jóvenes.

Por su lado, la evolución y la adaptación de la normativa sanitaria en los últimos años, también explica en gran parte la valoración positiva que los actores entrevistados manifiestan sobre la actual oferta y disposición de los servicios sanitarios «rurales». Sobre todo, por el avance que supuso para la sociedad rural pasar de un sistema de Asistencia Pública Domiciliaria ${ }^{9}$, apoyado en el ejercicio de unos pocos profesionales aislados - tanto espacial como organizativamente-, con escasos medios materiales y sin apenas descansos, a otro modelo más amplio e integrado, sustentado en el trabajo de equipos formados por múltiples y diferentes profesionales sanitarios ${ }^{10}$, coordinados entre sí, y dotados de más y mejores recursos (Escribano, 2009, p. 293).

${ }^{7}$ La etapa de Educación Secundaria Obligatoria contempla la enseñanza básica y abarca cuatro cursos académicos, entre los doce y dieciséis años de edad. Artículo 17.a. Ley 1/1990, de 3 de Octubre, de Ordenación General del Sistema Educativo. BOE n. 238 de 4 de octubre de 1990.

8 Aparte de este hecho, el traslado diario de alumnos que supone esta nueva organización educativa pierde gran parte del dramatismo anterior, gracias a medidas de apoyo como la generalización de comedores escolares; la ampliación del número de itinerarios y la reducción del tamaño de los mismos; el progresivo aumento y mejora de los sistemas de seguridad de los autocares escolares.

${ }^{9}$ El Decreto de 29 de septiembre de 1934 constituyó el Cuerpo de Médicos de Asistencia Pública Domiciliaria (APD) a partir de los funcionarios del escalafón del Cuerpo de Médicos Titulares Inspectores Municipales de Sanidad (Alonso, R., et al., 2000, p. 125).

10 Orden de 20 de noviembre de 1991, de la Conselleria de Sanidad y Consumo, por la que se establece el Reglamento de Organización y funcionamiento de los Equipos de Atención Primaria en la Comunidad Valenciana. DOGV n. 1.691, de 26 de diciembre de 1991. 
Obviamente, estos cambios también han repercutido favorablemente sobre las labores del personal sanitario asignado a centros rurales; no sólo porque estos ven incrementar y mejorar sus medios de trabajo, sino porque su actividad profesional deja de realizarse de forma aislada, una de sus principales reivindicaciones. De hecho, su consecución ha permitido acceder a otra serie de ventajas, entre las que destacan especialmente dos: por un lado, la separación entre la vida profesional y la vida personal, gracias al aumento registrado en el número de profesionales capaces de hacerse cargo de las labores sanitarias. Y por otro, poder centrarse estrictamente en el desarrollo de su oficio, en la medida que al aumentar la tipología de personal asignado a los centros sanitarios se consigue liberar a los médicos «rurales» de las tareas igualmente necesarias para el funcionamiento de estos servicios — gestión administrativa, mantenimiento, etc.- , pero no destinadas en principio a ser atendidas por ellos.

No obstante, el cambio en la percepción social sobre el nivel de servicio y la dotación existente en materia educativa y sanitaria elemental, no procede únicamente de la evolución y la adaptación normativa sectorial a las especificidades del medio rural. Es más, con frecuencia la mayor parte de los entrevistados hacen referencia a factores de tipo espacial, cuya importancia llega a ser incluso más elevada al actuar directamente sobre aspectos tan sensibles como la proximidad, la accesibilidad y la movilidad, y por tanto favorecer el acceso y el uso de los equipamientos y los recursos asociados a ambos servicios básicos (Entrena, 2006, p. 193).

Así, por ejemplo se gana en proximidad gracias a que servicios y profesionales hasta ahora centralizados en unidades jerárquicas superiores - como los hospitales-, se desplazan por determinados periodos de tiempo hasta centros localizados en el medio rural, coordinando a la vez su labor entre ellos. También se mejora la proximidad a través de la firma de convenios interterritoriales entre distintas administraciones para el uso de servicios similares, y de este modo mejorar en la práctica las zonificaciones regionales poco operativas. O simplemente, mediante el aumento del número de equipamientos e instalaciones educativas y sanitarias existentes en estas zonas.

Las actuaciones en materia de accesibilidad, desde el aumento y mejora de las infraestructuras de comunicación, hasta las condiciones o normas que regulan el funcionamiento de los servicios - como horarios de apertura, libertad para la elección de médico, citación telefónica, etc.- son señaladas también como básicas para el desarrollo de estos territorios y el consecuente aumento de su atractivo (Escalona y Díez, 2007, pp. 66, 87 y 89).

Aunque de las tres variables espaciales, la movilidad es la más determinante si tenemos en cuenta que ofrece no sólo mayores perspectivas de inte- 
rrelación territorial, sino que además sus posibles actuaciones necesitan con frecuencia de «menores» asignaciones económicas que la mayor parte de iniciativas de las otras dos variables. Un buen ejemplo, procede del aumento registrado por parte de colectivos que actúan sin ánimo de lucro, pero con una clara voluntad por completar la, en ocasiones «limitada» oferta de transporte público sanitario.

Ahora bien, tampoco hay que olvidar que la percepción relativamente positiva existente en los territorios analizados sobre la situación en la que los servicios básicos de educación y sanidad se organizan, es resultado de la cada vez mayor conciencia que la sociedad rural tiene sobre las limitadas posibilidades que su reducido tamaño demográfico les confiere, y la consecuente dificultad de conseguir más equipamientos e infraestructuras de todo tipo (Furuseth, 1998, pp. 236-238). En consecuencia, la población de estas zonas se encuentra en parte acostumbrada a la situación actual —a sus ventajas e inconvenientes-, buscando eso sí destacar siempre las primeras; de ahí que a menudo, se señalen las fortalezas que estos servicios ofrecen en comparación a los de otras zonas: menores problemas de tráfico, contacto más personalizado y próximo con sus profesionales, etc.

En definitiva, nos encontramos ante tres tipos de variables - normativas, espaciales y actitudinales - que explican en gran medida la percepción positiva que en general tiene la población rural, sobre la oferta y la organización que presentan los servicios educativos y sanitarios de carácter elemental en sus ámbitos de vida cotidiana. Aun así, como señalábamos antes, esto no impide que en el conjunto de los espacios rurales existan aspectos a mejorar; sobre todo, en aquellos municipios con una infraestructura educativa y/o sanitaria relativamente peor en cantidad, tipología y capacidad de atención es decir, en las localidades de menor tamaño demográfico- (Escribano, 2010, pp. 36-43). En consecuencia, es lógico que para estas poblaciones el papel que estos servicios desempeñan en la calidad de vida se valore, como veremos a continuación, de forma distinta.

LA INFLUENCIA DE LOS SERVICIOS EDUCATIVOS Y SANITARIOS BÁSICOS SOBRE LA CALIDAD DE VIDA DE LA SOCIEDAD RURAL

En líneas generales, las argumentaciones ofrecidas por los entrevistados sobre los principales componentes que la sociedad rural estima responsables de su calidad de vida, y en particular el valor que en ella tienen los servicios educativos y sanitarios elementales, nos indican la existencia, primero, de dos si- 
tuaciones según el papel más o menos activo que se les concede a estos coincidiendo cada una de ellas con los dos conjuntos de municipios trabajados; por un lado los de mayor población, y por otro los menos poblados- Y segundo, para el conjunto de municipios en donde los servicios educativos y sanitarios presentan mayor valoración, se llegan a observar diferencias en la capacidad de intervención de cada uno de ellos (figura 3).

\section{FIGURA 3}

\section{EL PAPEL DE LOS SERVICIOS PÚBLICOS EDUCATIVOS Y SANITARIOS EN LA CALIDAD DE VIDA RURAL}

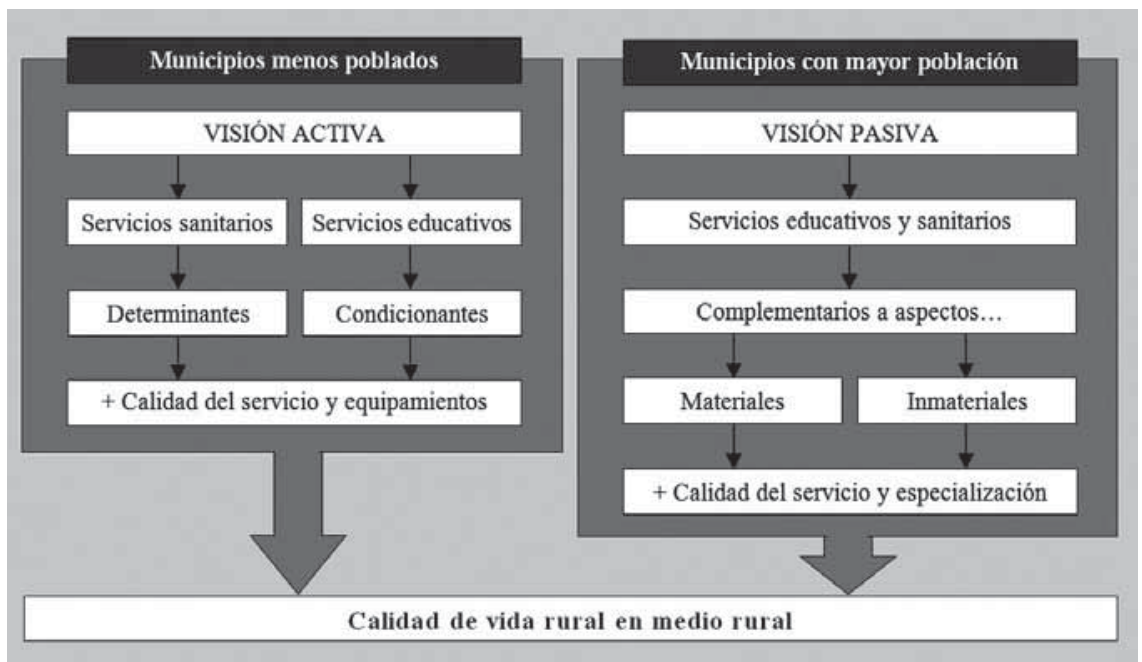

Fuente: elaboración propia a partir de los resultados de las entrevistas realizadas en los años 2006 y 2007.

Cuando los servicios educativos y sanitarios son caracterizados por su capacidad para influir de manera activa en la calidad de vida del medio rural, nos situamos en los municipios con menor población. Sencillamente, porque para los habitantes de estas localidades disponer de estos servicios en el mismo municipio de residencia supone un factor fundamental para permanecer en ellos, y por tanto de bienestar, al igual que la movilidad individual de que pudieran disfrutar — mediante automóvil particular_, la oferta laboral en proximidad, la vivienda en propiedad, y las relaciones sociales y familiares locales (Escribano, 2011, pp. 12-20). 
Tres son las principales razones que nos ayudan a entender la importancia de los servicios educativos y sanitarios básicos en estos municipios: en primer lugar, por la negativa social a perder una clase de servicios que desde siempre han estado presentes en estas zonas, y que tras los procesos de despoblación acontecidos en los últimos años, no sólo son los últimos en permanecer abiertos, sino que además constituyen casi la única vía de interacción social. Es decir, se trata de una clase de servicios básicos que junto a otros como las pequeñas tiendas locales de alimentación, las oficinas de correo, los bares, etc., constituyen el «pegamento» de la vida rural. Por ello, las señales de cierre y supresión de estos son en realidad amenazas al conjunto de comunidades rurales y a su modo de vida en general, ya que su desaparición no sólo supone una pérdida de funciones elementales, también conlleva el deterioro de uno de los rasgos simbólicos más importantes del mundo rural (Woods, 2006, pp. 586-589; Jean y Champollion, 2007, pp. 24-26; Bustos, 2009, pp. 457-458; Brereton et al., 2011, pp. 220-221).

En segundo lugar, puesto que con frecuencia los grupos de población más numerosos en estas zonas no disponen y/o no pueden hacer uso de medios de transporte particulares con los que desplazarse de forma autónoma de un núcleo a otro - es decir, a través del automóvil particular-, si estos servicios terminan por localizarse fuera del ámbito local su calidad de vida se reduce debido a la necesidad de recurrir a un transporte público caracterizado por su limitada oferta y escasa ordenación. En consecuencia, su «penalización rural» se ve incrementada al tener que hacer frente a unos tiempos de acceso a los servicios mayores; modificar sus hábitos de uso —no se pueden utilizar realmente cuando son necesarios-; cambiar la organización de su vida cotidiana, debido a la dificultad para compaginar su acceso y/o su uso con otro tipo de tareas habituales - como por ejemplo, la compra de productos de primera necesidad-; etc. (Hernández y Moltó, 2002, pp. 660-662; Collantes, 2005, pp. 528-529; Fundación Encuentro, 2007, pp. 388-389; Brereton et al., 2011, pp. 220-221).

$\mathrm{Y}$ en tercer lugar, porque la alternativa de recurrir a las prestaciones de municipios contiguos supone el uso de unas vías de comunicación con frecuencia no sólo en «mal» estado de conservación y con trazados estrechos y sinuosos - resultado de una abrupta orografía-, sino también inutilizadas durante largos períodos de tiempo debido a problemas de viabilidad invernal (Escalona y Díez, 2007 , pp. 67, 72, 103-104). En consecuencia, la inseguridad de desplazarse por ellas hará que su uso se reduzca al mínimo, o bien que determinada población evite utilizarlas trasladando su domicilio habitual a otros núcleos en los que estos servicios se encuentren asegurados físicamente, y sean accesibles sin necesidad de recurrir a ningún medio de transporte (Escribano, 2011, p. 22). 
Ahora bien, pese al destacado valor ofrecido tanto a los servicios educativos como a los sanitarios, la capacidad de intervención en la calidad de vida con que los entrevistados juzgan a ambos en los municipios menos poblados, es un tanto desigual a favor de los de carácter sanitario. Básicamente, debido al proceso de envejecimiento y «vacío» demográfico que afecta de forma más clara a estas localidades, ya que en ellas la emigración de población joven y adulta ha actuado con frecuencia de forma más temprana y continua, frente a los procesos de emigración de zonas demográficamente más dinámicas en donde estos son relativamente recientes, de carácter más intermitente, y/o compensados en cierta parte por la llegada de «nuevos» residentes. En consecuencia, en los núcleos con menor población quedan cada vez menos habitantes y, en cierta forma, estos se encuentran socialmente más aislados ante la falta de lugares de interacción social — debido a su cierre por escasa rentabilidad económica - y/o de población con la que relacionarse.

De este modo, el matiz que hace que los servicios sanitarios de carácter elemental aparezcan como los responsables directos de la calidad de vida rural en estos municipios, es la función de relación social y de apoyo psicológico que ofrecen a la población más abundante en los mismos. Es decir, su valor excede la mera función asistencial con que son concebidos normativamente, convirtiéndose así en un punto focal para la vida comunitaria, gracias por ejemplo a las conversaciones informales que surgen a su alrededor y que estructuran la vida social de la localidad. Por tanto, la simple dicotomía de disponer o no de servicios sanitarios es ya sinónimo de disfrutar o no de cierta calidad de vida, sin necesidad de llegar a plantearse cualquier otro tipo de variación en la atención recibida que pudiera aumentar y/o disminuir dicho bienestar, como la amplitud de horarios, las posibilidades de transporte a demanda, etc.

No obstante, la menor relevancia que alcanzan los servicios básicos educativos en estos municipios no hay que confundirla con desinterés. Se trata más bien, de una cuestión vinculada a la composición de la demanda existente, la capacidad de que dispone ésta para hacer prevalecer sus necesidades, y sus características. De hecho, la población capaz de beneficiarse de los servicios educativos presenta normalmente una mayor facilidad de desplazamiento que la población envejecida, gracias a sus mejores condiciones de salud y a la diversidad de transportes disponibles de los que hacer uso, desde medios familiares hasta otros de carácter público como los autocares escolares.

En consecuencia esta población puede adaptarse mejor al espacio, y por tanto depender menos de la oferta local, lo que se traduce en dos resultados: por un lado, disminuye la valoración de estos servicios como factores determinantes de la calidad de vida. Simplemente con estar disponibles en un entorno 
cercano — comarcal y/o mancomunado-, permiten disfrutar a sus usuarios de otros aspectos y valores capaces de mejorar y/o incrementar su bienestar diario. Sobre todo, gracias a la sensación de tiempo y libertad para conciliar la vida laboral con la de tipo familiar, o sencillamente poder realizar otras actividades que acrecientan la percepción de utilidad y/o productividad de las acciones diarias (Michalski y Marland, 2001, p. 17; Woods, 2006, p. 588; Shucksmith et al., 2009, pp. 1.283-1.285).

Y por otro, favorece introducir el tema de la calidad de los equipamientos y servicios como aspecto igualmente capaz de intervenir en la calidad de vida de la sociedad rural (Auh y Cook, 2009, p. 380). De hecho, es habitual que cada vez más padres empleen aspectos relacionados con el tipo y la cantidad de la oferta escolar y extraescolar, la proximidad y la actitud de los docentes con la sociedad local, sus competencias pedagógicas, su estabilidad laboral, etc., para decidir la escuela en la que matricular a sus hijos ${ }^{11}$ (Michalski y Marland, 2001, p. 17; Bouju-Goujon, 2007, pp. 84-88, 91-104; Walker y Clark, 2010). Es decir, para ciertos entrevistados contar simplemente con estos servicios resultará «insuficiente» si los mismos sólo se ofertan en términos de cantidad y no se atiende a las cualidades que los configuran: desde los materiales con que se trabaja en ellos, hasta su disposición espacial, capacidad adaptación a las demandas sociales o implicación de sus profesionales.

Por su parte, en los municipios con mayor número de habitantes la situación cambia considerablemente, puesto que como nos trasladan los entrevistados, en ellos los servicios educativos y sanitarios básicos apenas son valorados por su capacidad para incidir sobre la calidad de vida rural. Para la población local se trata de unos servicios elementales que con dificultad van a desaparecer de sus municipios debido a la elevada demanda con la que cuentan, que no sólo asegura la dotación y cobertura básica de ambos, sino que también permite disponer tanto de niveles más especializados como de otra serie de prestaciones sociales, culturales, económicas, etc. En consecuencia, su valoración en la calidad de vida pasa por completo desapercibida, siendo otros los elementos que condicionan el bienestar diario de que se disfruta en estas zonas. De hecho, en líneas generales estos pueden diferenciarse en dos grandes conjuntos complementarios, según se incida en aspectos materiales o no:

${ }^{11}$ Una de las consecuencias inmediatas que este cambio provoca es la alteración de los mapas escolares. Básicamente, porque los centros educativos con mayor capacidad para ofertar actividades extraescolares y/o complementarias, y calidad en sus recursos — plantillas estables, equipamientos actualizados a las necesidades educativas... - ven aumentado el número de alumnos en detrimento de aquellos otros sin las mismas capacidades (Escribano, 2011, p. 21).

Estudios Geográficos, Vol. LXXIII, 272, pp. 35-61, enero-junio 2012 ISSN: 0014-1496, eISSN: 1988-8546, doi: 10.3989/estgeogr.201202 
a) Por un lado, existe la percepción de que la calidad de vida en el medio rural procede básicamente de elementos vinculados tanto con el medio natural — relacionados con el clima y la disposición de sus recursos y paisajes resultantes - como con el espacio, entendiendo éste desde una doble perspectiva suplementaria:

- Como la disponibilidad de tierras para realizar algún tipo de tarea agrícola de autoconsumo - pequeños huertos—, o simplemente actividades de ocio como por ejemplo jardinería, que permitan desde recuperar y/o mantener recuerdos familiares y valores asociados a la ruralidad, hasta superar el modelo artificial de vida urbana y trabajo asalariado, incapaz de ofrecer algo más que pseudo-satisfacciones por el esfuerzo y el trabajo diario (Hervieu y Leger, 1980, p. 18).

- Y como vía para disponer de una vivienda en propiedad capaz de responder a un estilo y tipología concreta, habitualmente de baja densidad, y por tanto asegurarse así cierta privacidad.

Aunque también dentro de esta agrupación encontramos otro tipo de valores materiales, como por ejemplo: la disponibilidad de vehículo particular con el que desplazarse y hacer uso de los servicios del entorno, tanto socioculturales como medioambientales; la posibilidad de tener un trabajo adecuado al nivel de formación adquirido; o incluso, la opción de contar con buenas telecomunicaciones: cobertura móvil, acceso a internet a través de banda ancha, etc. (Fundación Encuentro, 2007, p. 384; Kazana y Kazaklis, 2009, pp. 214-215; Grgić et al., 2010, pp. 655-658).

b) Por otro lado, para una cantidad significativa de población residente en estos espacios, la calidad de vida deriva igualmente de los valores ambientales existentes en los mismos; las mayores posibilidades de disfrutar de éstos al estar menos afectados por la contaminación, polución y/o degradación lumínica, acústica..., propia de zonas urbanas; y del capital social que caracteriza a estas comunidades. De hecho, las redes sociofamiliares locales a través de las que la mayor parte de la gente se conoce entre sí, y en donde se generan valores como la amistad, la ayuda mutua, la sinceridad, o la simple idea de pertenecer a un grupo social concreto, son fundamentales no sólo en la atracción hacia la comunidad desde un punto de vista individual, sino también para influir en la satisfacción residencial (Auh y Cook, 2009, pp. 378-379 y 387388). Sobre todo, gracias a la cercanía social resultante del encuentro cotidiano y la realización de toda una serie de tareas habituales en un 
ámbito espacial reducido; es decir, en un medio donde el establecimiento de lazos de confianza y apoyo resulta más sencillo que en espacios complejos y con más población con la que interactuar.

Ahora bien, si examinamos específicamente la percepción que los actores entrevistados de los municipios con mayor población muestran sobre la influencia de los servicios educativos y sanitarios elementales en su calidad de vida, de nuevo resulta que son las prestaciones sanitarias las señaladas por su mayor capacidad para incidir en ésta (figura 4). Los principales argumentos son similares a los empleados anteriormente: primero, se trata de los servicios con mayor demanda potencial, al abarcar prácticamente a toda la población municipal, e incluso en algunos casos supramunicipal; pero también real, ya que si bien ésta se presenta en porcentajes más reducidos que en los municipios menos poblados, la población envejecida sigue siendo mayoritaria, sobre todo durante temporadas concretas como períodos estivales, vacaciones y ciertos fines de semana (Hernández y Moltó, 2002, pp. 662-663). Y segundo,

FIGURA 4

VALOR SOCIAL DE LOS SERVICIOS EDUCATIVOS Y SANITARIOS BÁSICOS EN LA CALIDAD DE VIDA SEGÚN MUNICIPIO Y SERVICIO ANALIZADO
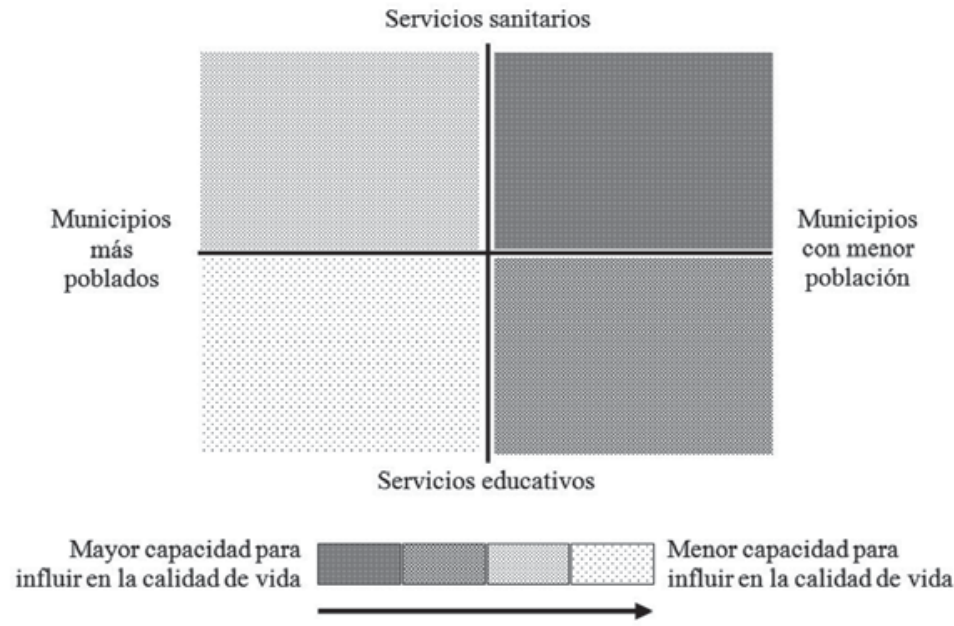

Nota: a mayor intensidad de la cuadrícula, mayor calidad de vida rural gracias a los servicios educativos y/o sanitarios.

Fuente: elaboración propia a partir de los resultados de las entrevistas realizadas en los años 2006 y 2007.

Estudios Geográficos, Vol. LXXIII, 272, pp. 35-61, enero-junio 2012

ISSN: 0014-1496, eISSN: 1988-8546, doi: 10.3989/estgeogr.201202 
sus usuarios más habituales son los que tienen mayores necesidades de disponer de ellos localmente, ante los frecuentes problemas que presentan en cuanto a autonomía personal y/o capacidad de desplazamiento —el problema de los transportes públicos afecta casi por igual a todos los espacios rurales-. De ahí que también sea normal encontrarnos con un mayor número de solicitudes por ampliar la oferta sanitaria que caracteriza a estas localidades, y que vienen a reforzar la mayor relevancia que para la población local tienen, o pueden llegar a tener, estos servicios en la calidad de vida.

\section{REFLEXIONES FINALES}

Si tenemos en cuenta la situación educativa y sanitaria elemental en la que se hallaba la práctica totalidad de territorios rurales durante el último cuarto del siglo pasado, es lógico encontrar que la sociedad rural en general valore de forma positiva tanto el tipo como la cantidad de oferta de ambos servicios disponible en la actualidad. De hecho, su mejora ha supuesto un incremento directo tanto de la calidad de vida de estos espacios como de las perspectivas y situaciones desde las que la población rural disfruta de ésta.

No obstante, esta pluralidad de opciones hace ahora que la capacidad de ambos servicios para incidir en el bienestar diario de la población no sea vista de idéntica forma por todo el medio rural. Así es como encontramos, por un lado, actitudes para las que contar tanto con los servicios educativos como sanitarios resulta clave para alcanzar y/o mantener una calidad de vida adecuada; y otras para las que, por otro lado, se trata de unos servicios sin apenas importancia, o que en el mejor de los casos tan sólo son capaces de incidir de forma secundaria en la calidad de vida. De hecho, así ocurre en los municipios con mayor población, al verse aquí como unos servicios asegurados gracias a la elevada demanda existente, y por tanto cuya oferta no corre riesgo de desaparición.

En todo caso, esto no quiere decir que se trate de unos servicios sin problemas y/o necesidades de mejora. Es más, el reciente viraje neoliberal del Estado de Bienestar y la tendencia resultante a la concentración de cada vez más servicios, entre ellos los educativos y sanitarios aquí analizados, demuestra no sólo que todavía hay margen de actuación, sino que los avances alcanzados hasta el momento pueden desaparecer a corto y/o medio plazo; entre ellos, gran parte de la calidad de vida de la que se disfruta actualmente en el medio rural.

Todo ello en un momento en el que el medio rural, primero, no demanda necesariamente un mayor número de servicios educativos y/o sanitarios —en 
líneas generales se acepta la cantidad de la oferta actual-, sino que éstos sean capaces de recoger tres tipos de actuaciones:

a) En primer lugar, que ambos servicios consigan adaptarse a las necesidades de la población más representativa de estos espacios, y evitar así problemas de desatención y/o de ineficiencia en el uso de los recursos.

b) En segundo lugar, que tanto unos como otros alcancen una mayor calidad, ya que ésta influye claramente en la mayor o menor utilización que la población hace de las prestaciones educativas y/o sanitarias. Por ejemplo, con frecuencia en zonas rurales nos encontramos cada vez con más grupos de población para los que relativamente poco o nada importa contar con estos niveles elementales en la proximidad de sus domicilios, si el servicio que prestan es concebido de insuficiente y/o inadecuado, y necesitan buscar otros puntos en los que poder acceder a una oferta similar pero caracterizada por una mayor y/o mejor calidad.

c) Y en tercer lugar, que ambos servicios se ajusten realmente a la idiosincrasia de estos territorios, tanto desde el punto de vista de la actitud de la sociedad rural como de su organización espacial —a menudo, caracterizada por su elevada dispersión-. Un objetivo para el que se proponen a su vez tres líneas de trabajo complementarias:

- El incremento de la proximidad a estos dos servicios mediante actuaciones en la variable más determinante para conseguir el uso eficiente de los mismos, pero a la vez más deficitaria e insatisfactoria: la movilidad derivada del uso de transportes públicos (Escalona y Díez, 2007, p. 111).

- El ajuste, en la medida de lo posible, de la organización funcional de ambos servicios a los estilos de vida de la población local; por ejemplo, a través de la adopción de horarios de atención sanitaria general coordinados con la duración de las jornadas de trabajo predominantes en estas zonas (Bustos Gisbert, 2005, pp. 148-149).

- El desarrollo de un marco normativo integral capaz de superar la visión sectorial de las distintas políticas existentes en materia de servicios a la población. En particular, por la configuración universalista que tradicionalmente las ha caracterizado - es decir, sin distinción alguna entre zonas urbanas y rurales-; su falta de adaptación a las diferentes particularidades sociales, resultado de su voluntad por generar respuestas homogéneas; y su confección incapaz de ofrecer soluciones integrales a las nuevas demandas sociales, precisamente cuando esto sería lo más demandado (Fundación Encuentro, 2007, pp. 391-392). 
Segundo, en un momento en el que los servicios educativos y sanitarios elementales se presentan como uno de los recursos más importantes en los procesos de autoafirmación de la identidad local, de búsqueda de raíces y de referencias tangibles, de cercanía y de proximidad, frente el avance de la uniformidad y la homogeneidad urbana (Entrena, 2006, pp. 180-184 y 200; Bustos, 2009, pp. 451 y 455). De hecho, una de las potencialidades más reconocidas de estos servicios, y en concreto de la escuela «rural», es su capacidad para ofrecer a la sociedad rural toda una serie de estrategias y recursos con los que poder entender y respetar la cultura local, valorar las fiestas tradicionales, el entorno natural, la propia historia del pueblo, etc.; es decir, ofrecer una estructura abierta a partir de la que crear, conservar y difundir conjuntamente todo una serie de conocimientos locales (Boix, 2003, pp. 4-7).

Y tercero, en un momento en el que la cada vez mayor dependencia del automóvil particular está mostrando claramente sus aspectos negativos sobre la calidad de vida de la población en general, pero en particular sobre la residente en zonas rurales. Por un lado, a través de la degradación y/o desaparición que el uso de este medio de transporte genera progresivamente sobre toda una serie de recursos medioambientales de gran valor, presentes todavía en estos espacios en su estado natural, y que suponen por ello uno de sus principales atractivos. Y por otro, acelerando la exclusión de aquella parte de la sociedad rural más vulnerable a la desaparición de estos servicios, o concentración en el mejor de los casos (Brereton et al., 2011, p. 223). No sólo porque esta población ve aumentar los costes temporales de acceso a los mismos, sino porque también termina por experimentar una reducción de sus recursos económicos como resultado de la crisis económica actual, al aumentar ésta los precios de los combustibles fósiles, y disminuir a su vez las opciones laborales existentes.

De ahí que si los actores entrevistados estiman de forma positiva el papel desarrollado por estos servicios en la calidad de vida de la población rural, ésta es una valoración resultado en gran parte del esfuerzo y el coste con que los habitantes de estas zonas asumen por cuenta propia la resolución, o más bien la mitigación, de los problemas que afectan a estos servicios. Es decir, gracias a la inversión adicional que en aspectos materiales como los recursos económicos destinados al transporte, e inmateriales como el tiempo a invertir para acceder a las prestaciones, realizan tanto sus usuarios como los profesionales que trabajan en ellos —al aceptar por ejemplo, ámbitos territoriales más amplios de actuación, realizar tareas que les impidieran ser más eficientes, etc.-

Recibido: 22/08/2011

Aceptado: 03/04/2012 
BIBLIOGRAFÍA

Alonso, R.; Bertral, C.; García, L.; Fajardo, A.; Martín-Carrillo, P.; Ornia, S.; Reguant, M. y Vela, P. (2000): "El medio rural: una visión mirando al futuro (I)". Atención Primaria, 26/2, pp. 20-130.

Auh, S. y Cook, C. (2009): "Quality of community life among rural residents: an integrated model”. Social Indicators Research, 94/3, pp. 377-389.

Boix, R. (2003): "Escuela rural y territorio: entre la desruralización y la cultura local". Revista Digital eRural, educación, cultural y desarrollo rural, 1/1, 8 pp.

Bouju-Goujon, A. (2007): "Familles et écoles: pratiques et représentations", en Y. Jean (dir.): Géographies de l'école rural : acteurs, réseaux, territoires. París, Ophrys, pp. 79-106.

Brereton, F; Bullock, C.; Clinch, J. y Scott, M. (2011): "Rural change and individual well-being: the case or Ireland and rural quality of life". European Urban and Regional Studies, 18/2, pp. 203-227.

Bustos Gisbert, M. L. (2005): "Envejecimiento y despoblación: dos problemas básicos para la revitalización de la Sierra de Francia (Salamanca)". Boletín de la Asociación de Geógrafos Españoles, 40, pp. 135-152.

Bustos, A. (2009): "La escuela rural española ante un contexto en transformación". Revista de Educación, 350, pp. 449-461.

Collantes, F. (2005): "Declive demográfico y cambio económico en las áreas de montaña españolas, 1860-2000". Revista de Historia Económica, 23/3, pp. 515-540.

Conselleria de Agricultura, Pesca y Alimentación (2009): Programa de desarrollo rural de la Comunidad Valenciana 2007-2013. Valencia, Conselleria de Agricultura, Pesca y Alimentación, 581 pp.

Corchón, E. (2000): La escuela rural: pasado, presente y perspectivas de futuro. Barcelona, Oikos-Tau, 130 pp.

Deller, S.; Tsai, T.; Marcouiller, D. y English, D. (2001): "The role of amenities and quality of life in rural economic growth". American Journal of Agricultural Economics, 83/2, pp. 352-365.

Entrena, F. (2006): "Difusión urbana y cambio social en los territorios rurales: un estudio de casos en la Provincia de Granada". Revista de Estudios Regionales, 77, pp. 179-206.

Escalona, A. I. y Díez, C. (2007): "Despoblación territorial y oferta de servicios: diagnóstico y propuestas", en C. Serrano (coord.): Despoblación y territorio. Zaragoza, Centro de Estudios sobre la Despoblación y Desarrollo de Áreas Rurales, pp. 61-118.

Escribano, J. (2011): "El valor de los servicios educativos y sanitarios en los procesos de atracción y fijación de población en medio rural". Ager, Revista de estudios sobre despoblación y desarrollo rural, $41 \mathrm{pp}$.

Escribano, J. (2009): "Valoración social sobre la evolución de los servicios sanitarios en zonas rurales de la Provincia de Valencia y su impacto sobre la calidad de vida", en F. Pillet; M. Cañizares y A. Ruiz (coords.): Geografía, territorio y paisaje: el es- 
tado de la cuestión. Actas del XXI Congreso de Geógrafos Españoles. Ciudad Real, Universidad de Castilla-La Mancha, pp. 287-302.

Escribano, J. (2010): "Los servicios educativos y sanitarios en el desarrollo rural valenciano: principales problemas y posibles mejoras". Geographicalia, 58, $30 \mathrm{pp}$.

Esparcia, J. y Noguera, J. (2001): "Los espacios rurales en transición”, en J. Romero; A. Morales; J. Salom y F. Vera (coord.): La periferia emergente. La Comunidad Valenciana en la Europa de las regiones. Barcelona, Ariel Geografía, pp. 343-372.

Fundación Encuentro (2007): "Nuevos retos para los municipios rurales", en Fundación Encuentro (ed.): Informe España, 2007: una interpretación de su realidad social. El mundo rural en la encrucijada. Madrid, Fundación Encuentro, pp. 355-404. Disponible en: http://www.fund-encuentro.org/informe_espana/indiceinforme.php? $\mathrm{id}=\mathrm{IE} 14$ (Fecha de consulta: 09/04/2012)

Furuseth, O. (1998): "Service provision and social deprivation", en B. Ilbery (ed.): The geography of rural change. Londres, Longman, pp. 233-256.

Gómez Piñeiro, F. (2009): "Aproximación al sistema de indicadores de calidad de la vida urbana”. Lurralde. Investigación y espacio, 32, pp. 281-299.

Grgić, I.; Žimbrek, T.; Tratnik, M.; Markovina, J. y Juračak, J. (2010): "Quality of life in rural areas of Croatia: To stay or to leave?". African Journal of Agricultural Research, 5/8, pp. 653-660.

Hernández, M. y Moltó, E. (2002): "Los equipamientos y los servicios en las nuevas funciones de los espacios rurales. El ejemplo de la montaña de Alicante", en Universidad de Cantabria (ed.): Los espacios rurales entre hoy y mañana. Actas del XI Coloquio de Geografía Rural. Santander, Universidad de Cantabria, Santander, pp. 657-667.

Hervieu, B. y Leger, D. (1980): "Recours à la ruralité et crise". Economié rurale, 140, pp. 16-20.

Jean, Y. y Champollion, P. (2007): "Espaces ruraux français et écoles", en Y. Jean (dir.): Géographies de l'école rural : acteurs, réseaux, territoires. París, Ophrys, pp. 15-58.

Kazana, V. y Kazaklis, A. (2009): "Exploring quality of life concerns in the context of sustainable rural development at the local level: a Greek case study". Regional Environmental Science, 9/3, pp. 209-219.

Massam, B. (2002): "Quality of life: public planning and private living". Progress in Planning, 58/3, pp. 141-227.

Michalski, J. y Marland, R. (2001): "Dialogue avec les citoyens sur ce qu'ils jugent important pour la qualité de la vie au Canada : la lentille rurale", en Réseaux canadiens de recherche en politiques publiques. Otawa, Secrétariat rural. Agriculture et Agroalimentaire Canada, 66 pp. Disponible en : http://www.cprn.org/doc.cfm? doc $=48 \& \mathrm{l}=$ fr (Fecha de consulta: 18/08/2011).

Piqueras, J. (2012): Geografía del territorio valenciano. Naturaleza, economía y paisaje. Valencia, Universidad de Valencia, 256 pp.

Pospěh, P.; Delín, M y Spěšná, D. (2009): "Quality of life in Czech rural areas". Agricultural Economics, 55/6, pp. 284-295. 
Ray C. y Ward N. (2006): "The Futures of Rural Policy: The Significance of Rural Futures Studies". Discussion Paper. Centre for Rural Economy, 7, 11 pp.

Richmond, L.; Filson, G.; Paine, C.; Pfeiffer, W. y Taylor, J. (2000): "No-farm rural Ontario residents' perceived quality of life". Social Indicators Research, 50/2, pp. 159-186.

Royuela, V.; Lambiri, D. y Biagi, B. (2008): "Economía urbana y calidad de vida. Una revisión del estado del conocimiento en España”. Biblio 3W, Revista Bibliográfica de Geografía y Ciencias Sociales, XIII/794, 2008. Disponible en: http://www.ub.edu/ geocrit/b3w-794.htm (Fecha de consulta: 18/08/2011).

Shucksmith, M.; Cameron, S.; Merridew, T. y Pichler, F. (2009): "Urban-rural differences in quality of life across the Union European". Regional Studies, 43/10, pp. $1.275-1.289$.

Valles, M. (2002): Entrevistas cualitativas. Madrid, Centros de Investigaciones Sociológicas. Serie Cuadernos Metodológicos, 32, 195 pp.

Walker, M. y Clark, G. (2010): "Parental choice and the rural primary school: lifestyle, locality and loyalty". Journal of Rural Studies, 26/3, pp. 214-249.

Woods, M. (2006): "Redefining the "Rural Question": The new "politics of the rural" and social policy". Social Policy and Administration, 40/6, pp. 579-595.

\section{RESUMEN}

Una de las principales características del medio rural es su elevada calidad de vida; sin embargo, aspectos elementales en la estructuración de su sociedad como los servicios educativos y sanitarios básicos, apenas aparecen como responsables del bienestar del que goza su sociedad, aunque continuamente se apunte la necesidad de mejorar su oferta. En consecuencia, resulta interesante plantear cómo la población rural valora en realidad la capacidad de estos servicios para intervenir en su calidad de vida. Lógicamente, la diversidad de espacios rurales hace que los resultados difieran entre sí; de hecho, así lo demuestra el discurso de los actores clave entrevistados: mientras que para municipios poco poblados estos servicios resultan determinantes, sobre todo por la vertebración social que favorecen las prestaciones sanitarias, para aquellos otros con más habitantes apenas destacan sobre aspectos como el paisaje, el espacio, la tranquilidad..., por la presencia asegurada que su elevada población les garantiza.

PAlabras Clave: calidad de vida; escuela rural; centros sanitarios; movilidad; territorios rurales.

\section{AbSTRACT}

One of the main characteristics of rural areas is a higher quality of life. However, the basic aspects of the social structure, e.g. the basic education and health-care services, are barely responsible for the wellbeing of the society, although the necessity of improving their offer is continually emphasised. Consequently, it is interesting to dis- 
cover how the rural population actually values the capacity of these services to intervene in their quality of life. Logically, because of the diversity of spaces that constitute the rural areas, the results vary; at least that is what the analysis of the interviews of various key actors shows: while the services are crucial for the municipalities with low population, especially because the health-care services facilitate the social structure, for those with a higher population they barely stand out from other aspects, e.g. the landscape, space, tranquillity, etc., because due to a higher population they are guaranteed.

KEY WORDS: quality of life; rural school; health-care centres; mobility; rural territories.

\section{RÉSUMÉ}

Une des principales caractéristiques du milieu rural est sa qualité de vie élevée. Toutefois, des aspects fondamentaux dans la structuration de sa société comme les services éducatifs et sanitaires élémentaires ne sont pas presque signalés comme responsables du bienêtre. Par conséquent, il est intéressant d'examiner comment la population rurale évalué réellement la capacité de ces services pour intervenir dans sa qualité de vie. Logiquement, la diversité des zones rurales fait que les résultats soient différents ; de fait, ceci témoigne le discours des acteurs clés interviewés : tandis que pour les communes les moins peuplées ces services sont déterminants, spécialement pour la structuration sociale que les équipements sanitaires favorisent, pour les communes les plus peuplées ces services ressortissent assez peu comme facteurs de qualité de vie sur éléments comme le paysage, l'espace, la calme..., à cause de la présence assuré qui les permet une population élevée.

Mots CLÉs: qualité de vie; école rural; établissements sanitaires; mobilité; territoires ruraux. 